\begin{tabular}{l|l} 
Revista Educación \\
ISSN: 0379-7082 \\
ISSN: 2215-2644 \\
revedu@gmail.com \\
Universidad de Costa Rica \\
Costa Rica
\end{tabular}

\title{
El aprendizaje de la noción de objeto según la forma en niños de educación preescolar: Propuesta geometría en movimiento
}

Sobalvarro Chavarría, Laura Marcela; Camacho Álvarez, María Marta

El aprendizaje de la noción de objeto según la forma en niños de educación preescolar: Propuesta geometría en movimiento

Revista Educación, vol. 42, núm. 2, 2018

Universidad de Costa Rica, Costa Rica

Disponible en: http://www.redalyc.org/articulo.oa?id=44055139034

DOI: https://doi.org/10.15517/revedu.v42i2.28195

Esta obra está bajo una Licencia Creative Commons Atribución-NoComercial-SinDerivar 3.0 Internacional. 


\title{
El aprendizaje de la noción de objeto según la forma en niños de educación preescolar: Propuesta geometría en movimiento
}

\author{
The Learning of the Notion of Object According to The Form in Children of Early Childhood Education: Proposal \\ Geometry in Motion
}

Laura Marcela Sobalvarro Chavarría [1]

Colegio El Rosario, Costa Rica

laurasobalvarrochavarria@yahoo.es

Maria Marta Camacho Álvarez [2]

Universidad de Costa Rica, Costa Rica

mariamarta.camacho@ucr.ac.cr

\author{
DOI: https://doi.org/10.15517/revedu.v42i2.28195 \\ Redalyc: http://www.redalyc.org/articulo.oa?id=44055139034
}

Recepción: 13 Marzo 2017

Aprobación: 26 Abril 2018

\section{RESUMEN:}

Geometría en movimiento es una propuesta producto de una investigación cuyo objetivo principal fue analizar el efecto de la implementación de talleres que involucren juegos dirigidos al desarrollo de habilidades motrices y al aprendizaje de la noción de forma en la población infantil del ciclo de Transición de la Educación Preescolar (Sobalvarro 2015). El enfoque de investigación fue mixto, con predominio cuantitativo. Se aplicó un diseño cuasi experimental, específicamente de diseño inverso. El proceso estuvo organizado por siete momentos, cada uno constituido por una fase, así como una serie de actividades que generaban un producto. Los niños y niñas participantes estaban matriculados en el ciclo de Transición de la Educación Preescolar, del Colegio El Rosario, ubicado en San José, Costa Rica. El estudiantado eran de dos grupos, uno de ellos (constituido por 30 estudiantes) de participó en forma de grupo experimental y el otro (constituido por 31 estudiantes) en forma de grupo control, esta participación se hizo asignando aleatoriamente cada grupo. El tratamiento aplicado al grupo experimental consistió en implementar la propuesta, constituida de 10 talleres en los cuales se desarrollaron de 8 a 10 juegos, que incluían, en cada uno, contenidos motores y de noción de forma y, en momentos previamente determinados por el diseño, se hicieron las mediciones correspondientes. Los resultados demostraron que participantes del grupo experimental obtuvieron un efecto significativo en su aprendizaje en comparación con el grupo control en cuanto al tema de noción de forma. Se concluye que aprender noción de forma por medio de la propuesta Geometría en movimiento genera un aprendizaje mayor en comparación con una metodología en la que el movimiento no es el eje transversal.

Palabras clave: Educación preescolar, educación física, geometría.

\section{AbstraCt:}

This study analyzes the effect of targeted game workshops aimed at developing motor skills to help teach preschoolers about the notion of shapes. The subjects involved in the study were children currently enrolled in the Transition Cycle of the Costa Rican Preschool Education System (Sobalvarro 2015). Research conducted for this study was based on mixed methodologies with a predominantly quantitative focus and a quasi-experimental inverse design approach. The study structure consisted of seven

\section{NoTAS DE AUTOR}

[1] Maestría Ciencias del Movimiento Humano Universidad de Costa Rica; Entrenadora Gimnasia Artística Femenina; Participación Work and Travel Orlando, Florida, USA; Administradora Técnica Escuela Permanente de Gimnasia UCR; Directora Nacional de Jueces de la Asociación Costarricense de Gimnasia. Profesora de Educación Física y Deportes (2004 - 2017); Coordinadora Departamento de Educación Física y Deportes Colegio El Rosario; Delegada Congreso Panamericano de Educación Física y Deportes; ha participado en diversidad de cursos nacionales e internacionales para jueces de gimnasia artística femenina; Juez Campeonato escolares, nacionales e internacionales de gimnasia artística. Expositora en congresos nacionales de Educación Física, en Jornadas de Investigación y en Simposios de Educación Física y Deportes, Universidad de Costa Rica.

[2] Profesora catedrática de la Universidad de Costa Rica, doctorado en Administración Educativa, Exdirectora de la Escuela de Formación Docente y de la Feria Nacional de Ciencia y Tecnología. Responsable de las investigaciones: Mejoramiento del Proceso de enseñanza-aprendizaje de la matemática en la Educación Preescolar, vista desde la perspectiva de la Formación Docente; La Educación Preescolar en la era digital: retos y desafíos y Propuesta interdisciplinaria para el diseño de aplicaciones informáticas para dispositivos móviles que posibiliten mejorar los procesos de enseñanza y aprendizaje de la trigonometría de noveno año, PIIDE (CONARE). Coordinadora de diversos proyectos de acción social de la UCR dirigido a niñez en riesgo. Publicaciones: La integración del niño sordo en Preescolar; Sumar, restar, multiplicar, dividir es divertido. 
phases and a series of activities with end-results or outputs. The children involved in the study were enrolled in the Preschool Transition Cycle at Colegio El Rosario (El Rosario School) located in San Jose, Costa Rica. The students were grouped together into two randomly assigned groups - an experimental group (30 students) and a control group (31 students). The students in the experimental group attended 10 workshops which involved playing a series of 8 to 10 games. Each of the games were designed to develop motor skills and help the students understand about the concept of shapes. The corresponding metric assessment was made and recorded at predetermined timeframes. The results showed that the participants in the experimental group obtained significantly higher results compared to the control group with regards to their understanding of shapes. Thus, it can be concluded that learning about shapes through Geometry in Movement was more effective than learning through a methodology that does not incorporate movement.

KEYWORDS: Preschool education, Physical education, Geometry.

En el desarrollo del aprendizaje del ser humano, las matemáticas son una de las áreas del conocimiento que presentan mayores dificultades, debido a factores diversos, algunos de los cuales se mencionan con mayor regularidad son el desarrollo de métodos que limitan al estudiantado a memorizar o aquellos que están alejados de la cotidianidad, o son abstractos y superficiales (Calderón, 2008; Godino, Batanero y Font, 2003; Ruiz, 2000). Por ello se realizó la investigación denominada Geometría en movimiento: Efecto de su aplicación sobre el aprendizaje de la noción de objeto según la forma, para el ciclo de Transición de la Educación Preescolar, cuyo objetivo principal fue analizar el efecto de la implementación de talleres que involucren juegos dirigidos al desarrollo de habilidades motrices y al aprendizaje de la noción de forma en la población infantil del ciclo de Transición de la Educación Preescolar del Colegio El Rosario.

\section{Antecedentes}

Entre las investigaciones que anteceden a este objeto de estudio se encuentran la de Fernández, Obando, Rodríguez, Salazar y Trejos (2007), quienes evaluaron los conocimientos matemáticos y de relaciones sociales manifiestas por los niños y niñas, según los criterios que presenta el programa del ciclo de Transición del Ministerio de Educación Pública de Costa Rica (1996). Como principal conclusión del estudio se indicó que el uso de la tecnología, específicamente del material digital, no inhibe la construcción de los conocimientos, ni el establecimiento de las relaciones sociales en esta población estudiantil. Por su parte, Calderón (2008) planteó el objetivo de diseñar una propuesta curricular en línea para la enseñanza de la geometría, dirigida a estudiantes de sétimo año. Las categorías de la investigación se dividieron en tres bloques: la relación con la mediación pedagógica, la planificación prescrita y ejecutada y, el uso de nuevas tecnologías.

En la misma línea de propuestas metodológicas, Núñez, López, Quesada, Pineda y Solano (2008) realizaron un trabajo de investigación en el que participaron docentes y estudiantes de II ciclo de escuelas públicas del sistema educativo costarricense. Se efectuó un análisis descriptivo, y se llegó a determinar que el estudiantado, en el momento del estudio, no tenía una comprensión real de la geometría debido a que no poseía una construcción del conocimiento. El personal docente impartió mucha teoría y no hizo muchos ejercicios prácticos, no existió la secuencia de complejidad en el aprendizaje y las orientaciones metodológicas no eran las más adecuadas.

En trabajos específicos de movimiento y matemática, se encuentra a Serrano, Azofeifa y Araya (2008), quienes trabajaron con niños y niñas del ciclo de Transición de Educación Preescolar. El objetivo de esta investigación fue determinar los efectos cognitivos de dos grupos de una población infantil, uno experimental, al que se le aplicaron actividades físico-recreativas relacionadas con las matemáticas y el otro grupo, control, donde se emplearon actividades lúdicas que no tuvieran relación con la matemática. Concluyeron que existieron efectos significativos en el aprendizaje de la población infantil al aplicar 
actividades físico-recreativas relacionadas con la matemática y que la educación física puede ser un instrumento muy eficaz, utilizado para el aprendizaje de otras materias académicas.

Diferentes estudios indican que el movimiento permite a la población infantil realizar acciones continuas que posteriormente les darán la oportunidad de lograr procesos cognitivos más ajustados (Medrano, 2001; Piaget, 1975; Piaget, 1985; Oña, 1994; Ruiz et al., 2003; Verlee, 1986; Wallon, 1978). Mencionan y demuestran que es muy importante trabajar con infantes una metodología educativa con un alto componente motor.

Según Ruiz et al. (2003), las habilidades motrices tienen que ver con la organización y la clasificación del movimiento, lo que permite ver la motricidad como un todo en el desarrollo del ser humano y, a su vez, como herramientas útiles para otros aprendizajes.

Existen investigaciones educativas que establecen la relación entre juego- movimiento y el desarrollo de las capacidades integrales, entre ellas las cognitivas, afectivas y sociales. En este sentido, varias aluden a que las personas en la etapa educativa infantil tienen una inteligencia esencialmente práctica que pasa por el dominio de lo motor, punto desde el cual se deben construir los aprendizajes Blández, 2000; Medrano, 2001; Molina, 1990; Quirós y Arráez, 2006; Viciana, 2000).

Desde esta perspectiva, toda metodología basada en el principio del movimiento intentará centrarse en la utilización del cuerpo visto como una herramienta de primera mano, para generar un mayor aprendizaje en otras áreas como, por ejemplo, en las matemáticas.

Cabe mencionar que, si bien existe información relacionada con la enseñanza de la matemática en el área de la geometría, son pocas las investigaciones que establecen relación de la matemática con la educación física, concretamente en el tema de habilidades motrices, por lo tanto, estos temas fueron objeto de estudio de la investigación que origina este artículo.

Además, la mayoría de las investigaciones que se han realizado en el área de la matemática están enfocadas a los niveles de educación básica y la diversificada, mas no en la preescolar. Este ciclo se considera una etapa crucial de la educación en la cual la población infantil está más receptiva a aprender y, por ende, permite que se construyan las bases de todo aprendizaje futuro.

\section{Referentes teóricos}

Las investigaciones que se han realizado en el área de las matemáticas, en su mayoría están enfocadas en los niveles de educación básica y en la diversificada, mas no en la educación preescolar. Este ciclo se considera una etapa crucial de la educación, pues la población infantil está más receptiva a aprender y, por ende, permite que se construyan las bases del aprendizaje futuro. La etapa de 0 a 6 años es una etapa trascendental en la vida del ser humano. En este período, los aprendizajes son más rápidos y efectivos, por lo que la utilización de estrategias lúdicas con materiales concretos permite generar experiencias significativas para el estudiantado.

En los postulados propuestos por Jean Piaget, en el área de la psicología aplicada a la educación, es común encontrar que el movimiento sea considerado la base del aprendizaje. Esta idea es retomada por muchos otros estudios Gabbard, 2012; Gallahue y Ozmun, 2003; Lleixa, 2000; Hardy, Reinten-Reynolds, Espinel, Zask, \& Okely, 2012; Piaget, 1975; Piaget, 1985; Oña (1994); Ruiz et alt., 2003; Verlee, 1986; Wallon, 1978) que sostienen que el dominio motor es la base para la construcción de aprendizajes. Así mismo Ruiz et al. (2003) indican que la educación física en la educación infantil debe tener como finalidad el desarrollo de habilidades motrices y ser un medio para trabajar el resto de contenidos curriculares. En cuanto a habilidades motrices, Conde y Viciana (1997) proponen una clasificación con base en el desarrollo de tres aspectos de la motricidad: control y conciencia corporal, locomoción y manipulación.

Estos autores señalan que los primeros movimientos que hace el ser humano son los reflejos, que son involuntarios, y representan la base de la motricidad. A partir de estos reflejos se hacen adquisiciones motrices que tienen su origen en los tres aspectos mencionados anteriormente. De esta forma, se encuentra que en 
control y conciencia corporal se incluyen todas aquellas habilidades referentes al dominio del cuerpo y su conocimiento: actividad tónico postural equilibrada (ATPE), esquema corporal, lateralidad, respiración, relajación y senso-percepciones.

Asimismo, la clasificación considera que la locomoción incluye los primeros movimientos voluntarios y habilidades tales como reptar, gatear, trepar, ponerse de pie; desplazamientos naturales como la marcha y la carrera; desplazamientos acuáticos; desplazamientos construidos y los saltos. En la tercera categoría, que denominan manipulación, se sitúan todas aquellas adquisiciones relacionadas con el manejo de objetos, tales como: alcanzar, tomar, agarrar, soltar, arrojar, atajar y, más adelante, movimientos como lanzar y recibir, los cuales ya requieren un grado de mayor dominio.

Todas las habilidades antes mencionadas se dan en un espacio-tiempo determinado que, relacionado con las habilidades, van a generar un mejor desarrollo de las destrezas. Esta situación fomenta la coordinación, que no es más que el avance adecuado de todas y cada una de las habilidades anteriores. Estas habilidades motrices se derivan posteriormente en habilidades genéricas y estas se subdividen en específicas, las cuales se trabajan cuando ya existe mayor grado de madurez motriz.

Otra definición de habilidades motrices es la propuesta por Velázquez y Martínez (2005), autores que describen este concepto como el mayor número de movimientos posibles, desarrollados por los niños y las niñas, que permitan ampliar su riqueza motriz como base de futuros aprendizajes. Estos mismos autores clasifican las habilidades motrices en: habilidades perceptivas, habilidades básicas, habilidades genéricas, habilidades específicas y habilidades especializadas.

Velázquez y Martínez (2005) también mencionan que las habilidades perceptivas se dividen en tres bloques: percepción corporal (todo lo que tiene que ver con conocimiento de control y el ajuste corporal, lateralidad, respiración y relajación), percepción espacial (todo lo referente a orientación y estructuración espacial) y, por último, la percepción temporal (tiene que ver con la velocidad, la frecuencia y la duración de las diferentes acciones). Por lo tanto, las habilidades motrices básicas son los movimientos fundamentales para la adquisición de otras habilidades (genéricas y específicas), las cuales son desplazamientos, saltos, giros, lanzamientos, recepciones y equilibrios.

\section{Aprendizaje de las nociones de geometría}

Al enseñar la geometría a la población infantil en la educación preescolar es muy importante recordar lo que menciona Mira (2001):

Se debe entender que no se trata de introducir el estudio métrico de las figuras, sino de sistematizar las experiencias que los niños y las niñas realizan de forma espontánea en su exploración del espacio que les rodea y de los objetos que tienen a su alcance, así mismo se inicia una reflexión de las características de las figuras. (p. 299)

Entonces, es fundamental que la metodología elegida para esta tarea tenga presente la necesidad del movimiento y de exploración que caracteriza a la infancia, así como las grandes posibilidades educativas que ofrece la actividad motriz.

Con respecto a la geometría, esta es una de las áreas de la matemática que permite al individuo entender y analizar la información que recibe día tras día de su entorno. Es decir, su desarrollo va a dotar a los niños y las niñas de herramientas para desenvolverse e interpretar el medio, ya que en esta área de las matemáticas, es fundamental analizar las formas, clasificarlas, idear transformaciones, componer figuras, conocer propiedades de los objetos y estudiar relaciones entre ellos.

La noción de objeto según la forma, de acuerdo con Camacho (2004), es el estudio de las propiedades y las relaciones formales de las figuras del plano y del espacio. Es la inducción al aprendizaje de las figuras geométricas, basada en el reconocimiento de distintas formas y cómo se relacionan estas formas con elementos u objetos comunes al entorno. Específicamente para la investigación que sustenta este artículo se trabajó con 
el reconocimiento de figuras planas como círculo, óvalo, triangulo, cuadrado, rectángulo, trapecio y rombo, y de polígonos de 5, 6,7 y 8 lados, y la identificación de características como área, perímetro, lados, vértices y ángulos en estas figuras.

\section{Matemática activa creativa}

Con el objetivo de promover aprendizajes significativos en el área de las matemáticas, se ha venido desarrollando una metodología llamada matemáticas activa creativa (MAC), la cual fue impulsada por la Dra. María Marta Camacho Álvarez y tiene como objetivo principal la innovación de la forma en que los niños y las niñas aprenden matemáticas. Este proyecto se desarrolla desde 1996, está inscrito en la Vicerrectoría de Acción Social de la Universidad de Costa Rica con el código ED-2425 y sus resultados se han divulgado e implementado en países como Nicaragua, Guatemala, México, Argentina, Bolivia, Sur África. Es reconocido por una red mundial de emprendedurismo social llamada Ashoka.

Araya (2008) indica que se entiende por matemática activa y creativa a los métodos, las técnicas y los procedimientos que el personal docente utiliza para orientar a los niños y la niñas en la construcción de nociones matemáticas en un ambiente rico en materiales y actividades dinámicas

Según Camacho (2004), matemática activa y creativa es una metodología participativa en la cual el personal docente, madres y padres de familia son los facilitadores, mientras que los niños y las niñas son entes activos de todo el proceso educativo. En MAC se promueve que la población estudiantil desarrolle un pensamiento lógico- matemático por medio de diferentes circunstancias que promuevan la autonomía, la independencia, la creatividad y la puesta en práctica de diversas actividades lúdicas para lograr que se generen y faciliten aprendizajes significativos por medio de juegos y actividades.

\section{REFERENTES MEtodológicos}

El enfoque de investigación es mixto (cualitativo- cuantitativo), con predominio del cuantitativo. El proceso metodológico se organizó en siete momentos que abarcan desde la fase preparatoria; el acercamiento teóricopráctico; la elaboración y puesta en práctica de la propuesta, el análisis y procesamiento de la información y el informe de investigación; a su vez, cada momento estaba constituido por una fase, así como una serie de actividades que generaban un producto.

azar ni se emparejan, sino que dichos grupos ya estaban formados antes del experimento.

Se aplicó un diseño cuasi experimental, en el cual según Hernández et al (2006), los sujetos no se asignan al azar ni se emparejan, sino que dichos grupos ya estaban formados antes del experimento.

Dentro de los diseños cuasi experimentales, el elegido para este estudio es el diseño inverso y se define de la siguiente manera: O1 O2 T1 O3 O4 T2 O5 O6. Se escogió este diseño debido a que se desea determinar el valor de la línea base (O1 y O2), evaluar el tratamiento (cambios entre $\mathrm{O} 2$ y O3) y evaluar un período libre de tratamiento $(\mathrm{O} 3$ hasta $\mathrm{O} 4)$, volver a evaluar el tratamiento $(\mathrm{O} 4$ hasta $\mathrm{O} 5)$ y evaluar la repetición del estado de no tratamiento (O5 hasta $\mathrm{O} 6$ ).

A su vez, para tener mayor control, también se aplicó el diseño de grupo control no equivalente, de modo que el diseño del estudio es el siguiente

\section{$\mathrm{O} 1 \mathrm{O} 2 \mathrm{~T} 1 \mathrm{O} 3 \mathrm{O} 4 \mathrm{~T} 2 \mathrm{O} 5 \mathrm{O} 6$}

\section{$\mathrm{O} 1 \mathrm{O} 2 \mathrm{O} 3 \mathrm{O} 4 \mathrm{O} 5 \mathrm{O} 6$}

El objetivo principal de este estudio fue demostrar el cambio provocado por el tratamiento o variable independiente, en la variable dependiente: el aprendizaje de la noción de forma. El grupo experimental recibió el tratamiento denominado Geometría en movimiento en el cual se desarrollan conceptos de noción de forma, a la vez que se trabajan las habilidades motrices en los niños y las niñas. Integrantes del grupo control 
recibieron las clases regulares, tal y como se sugiere en el Programa del Ministerio de Educación Pública y según criterio de la docente a cargo del grupo.

Los procedimientos generales del estudio fueron organizados en diversos pasos que van desde la aprobación del estudio; el consentimiento informado para los padres y las madres de familia; la asignación de grupo experimental y grupo control; las mediciones iniciales; la intervención o puesta en práctica de la propuesta; las mediciones intermedias y las finales, para luego realizar el análisis de datos.

Las técnicas utilizadas fueron la entrevista semiestructurada para lo cual se realizó una guía con base en los objetivos de investigación, el análisis de documentos y los talleres que involucran aspectos de habilidades motrices y de nociones matemáticas, cada uno con sus respectivas bitácoras de trabajo, las observaciones participantes, cuyos resultados fueron anotados también en bitácoras, y la medición del tratamiento registrada mediante listas de cotejo. La validación de instrumentos se realizó con el criterio de personas expertas. Durante el tiempo del estudio se realizaron alrededor de seis mediciones u observaciones a los grupos, con el objetivo de llevar a cabo el diseño de investigación.

Se utilizaron dos estrategias de análisis, para la parte cuantitativa se realizó un análisis estadístico con estadística descriptiva, se analizaron las frecuencias absolutas y porcentajes, dada la naturaleza nominal de las variables. También se utilizó estadística inferencial y análisis de regresión logística binaria. Todos los resultados se corrieron por medio del paquete estadístico SPSS versión 15.0. Para el análisis cualitativo, se aplicó la técnica de triangulación metodológica, en la que se retoman los resultados de los diferentes instrumentos, las anotaciones de las bitácoras y el sustento teórico.

El estudio se realizó con niños y niñas del nivel de Transición de Educación Preescolar del Colegio El Rosario, institución semioficial, es decir, que funciona como institución privada, pero que es subvencionada por el Estado costarricense.

\section{DisCUSIÓN DE RESULTADOS Y ANÁLISIS}

Cabe recordar que este análisis se centra en los resultados de la aplicación de la propuesta Geometría en movimiento, es decir, en el efecto de la implementación de talleres que involucren juegos dirigidos al desarrollo de habilidades motrices (actividad tónico postural equilibrada (A.T.P.E), esquema corporal, desplazamientos, respiración y lanzamientos) y al reconocimiento de figuras planas como círculo, óvalo, triangulo, cuadrado, rectángulo, trapecio, rombo, pentágono, hexágono, pentágono y octágono, así como la identificación de características como área, perímetro, lados vértices y ángulos, en la población infantil del ciclo de Transición de la Educación Preescolar.

En general, esta propuesta está constituida por diez juegos organizados de acuerdo con la siguiente estructura: número de taller, nombre del juego, objetivos, contenido matemáticos, contenido de habilidades motrices, desarrollo de la actividad, variantes y materiales.

Luego de su aplicación se percibe en los resultados que hubo diferencia significativa en el aprendizaje en el grupo experimental en comparación con el control. Se determina que en cuanto al círculo, todo el estudiantado participante del estudio ya dominaba esa figura, lo que indica que es una figura básica que se aprende desde edades muy tempranas. Con respecto a las figuras planas del óvalo y del triángulo se puede indicar que la población infantil ya lo había aprendido, pues no hubo significancia en el modelo de regresión. Sin embargo, se puede decir que los pocos niños y niñas que no sabían esas figuras geométricas, a raíz de recibir el tratamiento, o sea, la propuesta Geometría en movimiento, las pudieron llegar a aprender para así completar el 100\% de los sujetos que aprendieron el reconocimiento de estas figuras en el grupo experimental, no así en el grupo control.

Con respecto a las tres figuras antes mencionadas (círculo, óvalo y triángulo), los resultados no muestran evidencia de que el aprendizaje se haya dado por el tratamiento. Es decir, que el grupo infantil ya sabía o había aprendido anteriormente esas figuras. 
Además, existió regresión logística binaria significativa en todas las demás figuras geométricas y conceptos geométricos evaluados. En todos estos casos, la pertenencia al grupo experimental tuvo un efecto importante sobre el aprendizaje, mientras que las diferencias de sexo no constituyeron un factor relevante para explicar los resultados (salvo en el caso del reconocimiento del hexágono). Por lo tanto, los efectos de la participación en el programa sobre el aprendizaje de las figuras y conceptos geométricos valorados, fue similar para las niñas y los niños.

Detallando la interpretación de estos resultados y basándose en las razones de ventaja $[\operatorname{Exp}(B)]$, se puede afirmar que quienes participaron del grupo experimental (independientemente de su sexo), tuvieron 5,003 veces más probabilidades de aprender a reconocer el cuadrado, en comparación con quienes participaron del grupo control.

Asimismo, el estudiantado del grupo experimental tuvo 25,796 veces más probabilidades de aprender a reconocer el rombo; más de 8 mil millones de veces más probabilidades de aprender a reconocer el trapecio; 27,077 veces más probabilidades de aprender a reconocer el rectángulo; más de 13 mil millones de veces más probabilidades de aprender a reconocer el pentágono; más de 383 mil billones de veces más probabilidades de aprender a reconocer el hexágono (en este caso se encontró que, independientemente del grupo al que pertenecieran, las mujeres tuvieron más posibilidades de aprender a reconocer esta figura, esto al tener 211 millones de veces más probabilidades que los hombres); más de 7 mil millones de veces más probabilidades de aprender a reconocer el heptágono; más de 16 mil millones de veces más probabilidades de aprender a reconocer el octágono; 9 veces más probabilidades de aprender el concepto de área, pintándola; más de 524 mil millones de veces más probabilidades de aprender el concepto de área señalándola y 869,724 veces más probabilidades de aprender el concepto de perímetro, pintándolo.

En los demás conceptos (área reconocida kinestésicamente; perímetro señalándolo y reconociéndolo kinestésicamente; ángulo pintándolo, señalándolo y reconociéndolo kinestésicamente; vértice pintándolo, señalándolo y reconociéndolo kinestésicamente), quienes participaron en el programa tuvieron 100\% de probabilidad de aprender, mientras que quienes estuvieron en el grupo control tuvieron $0 \%$ de probabilidades de aprender.

En síntesis, la participación en el programa contribuyó significativamente al aprendizaje del reconocimiento del cuadrado, rombo, trapecio, rectángulo, pentágono, hexágono, heptágono y octágono y también contribuyó significativamente al aprendizaje de los conceptos de área, perímetro, ángulo y vértice.

Los resultados de los análisis estadísticos reflejan claramente que el grupo experimental mostró mayor dominio y aprendizaje sobre el reconocimiento de las figuras planas y sus características que el grupo control.

Este desarrollo permite resaltar la importancia de que los grupos infantiles reciban en su programa curricular la asignatura de Educación Física, pues el fin de esta disciplina es promover una educación por medio del movimiento, que a su vez, desarrolle las habilidades motrices y contribuya al desarrollo integral del estudiantado. También, apoya la idea de que en el nivel preescolar, las actividades deben centrarse en el desarrollo motor para aprender otros conocimientos de diferentes áreas.

Con respecto a las figuras de cuatro lados, en el caso del rombo, 27 niños y niñas del grupo experimental, de un total de 30, llegaron a dominar el aprendizaje de esta figura y los cambios se dieron siempre posterior a recibir el tratamiento. En el caso del trapecio y del rectángulo, el 100\% del grupo experimental lo llegó a aprender.

Por otro lado, en los polígonos de 5, 6, 7 y 8 lados, los porcentajes de aprendizaje, es decir, los niños y las niñas que llegaron a dominar e identificar esas figuras, siempre están por arriba del $75 \%$, en comparación con $0 \%$ del dominio o aprendizaje en el grupo control.

Posteriormente, en la parte de reconocimiento de las características de las diferentes figuras geométricas referentes al área, perímetro, ángulos, vértices y lados, los resultados son abruptos y muestran grandes diferencias significativas a favor del grupo experimental. 
Todos los resultados descritos anteriormente, así como la triangulación de los datos recopilados por medio de las observaciones, las listas de cotejo, las bitácoras elaboradas por las docentes (docente observadora y docente investigadora) y la teoría que sustenta el estudio, muestran claramente que el movimiento favorece diferentes aprendizajes y que el dominio motor es fundamental en la construcción de nuevos conocimientos, encontrando así una relación directa entre actividad motriz y actividad cognitiva, lo cual se evidencia el acierto de haber trabajado por medio de una metodología con un alto componente motor. De esta forma, se refuerza la idea de que la educación física en la educación infantil debe tener como finalidad el desarrollo de habilidades motrices y debe ser un medio para trabajar el resto del contenido curricular. Estos criterios han sido parte de los aportes de muchos estudios, entre ellos los de Piaget (1975); Wallon (1978); Piaget (1985); Verlee (1986); Oña (1994); Ruiz et al. (2003); Fairbrother (2010); Spessato, Gabbard, \& Valentini, (2013); Cohen, Morgan, Plotnikoff, Callister, \& Lubans, (2014).

Los resultados anteriores demuestran que la participación, al recibir el tratamiento por medio de la propuesta Geometría en movimiento, favorece el aprendizaje del reconocimiento de las figuras geométricas y sus características. Estos hallazgos apoyan el sustento teórico de matemáticas activa y creativa que menciona que, de acuerdo con el aprendizaje de figuras geométricas, (1) los niños y las niñas están en capacidad de aprender cualquier figura geométrica en edades tempranas y (2) se debe dar a esta población estudiantil la oportunidad de aprender más y no limitarla a unas pocas figuras geométricas, para que el aprendizaje sea según lo demanda su capacidad (Camacho, 2004).

En este sentido, Molina (1990) indica que el juego es fundamentalmente un medio de aprendizaje. A partir del juego se pueden transmitir a infantes aprendizajes que, de otro modo, no serían interesantes para esta población, ya que el juego es una actividad que le produce placer y, por tanto, estará en disposición a aprender todo lo que sea necesario para tener éxito en sus juegos.

Blández (2000) menciona que la actividad lúdica es un recurso especialmente adecuado en la etapa de la educación infantil. Es necesario romper la aparente oposición entre juego y trabajo, que considera este último asociado al esfuerzo de aprender y el juego como diversión ociosa.

Por otro lado, Gutiérrez, Bartolomé y Hernán (1997) afirman la importancia del juego en la etapa infantil como un recurso indiscutible. Según estos autores, el personal docente a cargo de la población infantil debería utilizar esta tendencia natural para planificar las actividades educativas. Esta situación se presenta clara en este estudio, ya que el planeamiento principal se basa en una actividad lúdica que incluye el desarrollo de una habilidad motriz y una característica de alguna figura geométrica. Con ello, se abarcan dos áreas en una misma actividad y se apoya el desarrollo de una educación globalizada. En este sentido, también se encuentra Calderón (2008), quien menciona que existe una relación entre la geometría y otras áreas del conocimiento humano, como el arte, la ciencia, la educación corporal, la danza, la música y el movimiento. Estas áreas permiten generar un mejor aprendizaje, el cual se pudo observar y se comprobó con los resultados de los diferentes análisis estadísticos de este estudio.

Estos resultados dan sustento al Programa Matemática Activa y Creativa tal y como lo mencionan otras investigaciones (Araya, 2008; Calderón, 2008; Camacho, 2004; Coto, 2002), en las que se indica que el programa permite obtener un aprendizaje significativo, favorecer diversas áreas de desarrollo, desarrollar dinamismo y creatividad en el aprendizaje, obtener una mayor comprensión de la matemáticas por medio de diferentes estrategias, desarrollar las destrezas kinestésica y promover un aprendizaje de forma activa y creativa.

Todos estos resultados también constatan lo indicado por Azofeifa y Serrano (2008), quienes concluyeron que existen efectos significativos en el aprendizaje de la población infantil al aplicar actividades físicorecreativas relacionadas con las matemáticas y, que la educación física puede ser un instrumento muy eficaz, utilizado para el aprendizaje de otras materias académicas. Además, que al usar el movimiento de experiencias guiadas se facilita un mejor aprendizaje en materias académicas (Araya, 2008; Calderón, 2008; Coto, 2002; Fernández et al., 2007; Gallahue, 2005). 


\section{Conclusiones}

Geometría en movimiento es una propuesta metodológica que integra procesos de movimiento para que los niños y las niñas construyan conocimiento del mundo que les rodea. Por medio de diversas experiencias corporales, la población infantil creo hábitos, actitudes, comportamientos y conocimientos de su cuerpo y la relación entre este, el espacio y los objetos, además de mejorar su aprendizaje en la noción de forma.

Se pudo establecer la existencia de un efecto significativo en el aprendizaje al implementar la enseñanza de noción de forma por medio de la propuesta Geometría en movimiento, resultado que dio beneficios a participantes del grupo experimental.

El uso de juegos que incluyeran habilidades motrices y conceptos de noción de forma constituyeron un factor muy importante y motivador que generó un aprendizaje mucho mayor que utilizar un método tradicional y pasivo para enseñar.

Se demuestra una relación causal existente entre el movimiento y su efecto en el aprendizaje de la noción de forma. Esta situación trae consigo implicaciones para la formación del currículo de educación preescolar, ya que las clases de Educación Física deberían ser incluidas en su programa y, a su vez, desarrollar contenidos para una educación global.

\section{RECOMENDACIONES}

Los resultados obtenidos invitan a determinar algunas recomendaciones, tales como:

Continuar llevando a cabo el desarrollo de la propuesta en centros educativos del nivel preescolar, tal y como se sigue haciendo en la institución donde se desarrolló la investigación, como parte de los efectos positivos alcanzados. Tomar en cuenta la relación que existe entre el efecto de la implementación de talleres que involucren juegos cuyo contenido sea de habilidades motrices y noción de forma, sobre el aprendizaje de la geometría en la población infantil del ciclo de transición de la educación preescolar.

Dar a conocer la propuesta Geometría en movimiento y los efectos de su aplicación a los diferentes entes de la educación, con el objetivo de que sus resultados creen conciencia y permitan hacer cambios a nivel curricular, específicamente en la educación preescolar y la educación física.

Dar a conocer la propuesta y sus resultados al personal docente de las áreas de educación física y educación preescolar principalmente, de manera que si la población estudiantil no tiene al alcance a recibir educación física, pueda desarrollar este programa y, a su vez, enseñar contenidos, habilidades y destrezas.

Incorporar en los programas de estudio de las carreras relacionadas con la educación preescolar y la educación física aspectos académicos, resultados de la investigación y de la propuesta.

Generar espacios espacios de convivencia y enriquecimiento interdisciplinarios, por medio de los cuales el personal docente en formación y en ejercicio pueda tener capacitaciones sobre temas y metodologías relacionadas con esta investigación.

\section{REFERENCIAS}

Araya, Y. (2008). Los encuentros infantiles de matemáticas activa y creativa: Impacto que generan en las instituciones educativas (Tesis de licenciatura). Universidad Estatal a Distancia. San José, Costa Rica.

Blández, J. (2000). Programación de unidades didácticas según ambientes de aprendizajes. Barcelona: INDE Publicaciones.

Calderón, C. (2008). Propuesta curricular en línea para los procesos de enseñanza-aprendizaje de la geometría de sétimo año (Tesis de maestría). Universidad de Costa Rica. Ciudad Universitaria Rodrigo Facio. San José, Costa Rica. 
Camacho, M. (2004). Mejoramiento del proceso de la enseñanza- aprendizaje de las matemáticas en la educación preescolar, visto desde la perspectiva de la formación docente. Ciudad Universitaria Rodrigo Facio. San José, Costa Rica.

Cohen, K. E., Morgan, P. J., Plotnikoff, R. C., Callister, R., \& Lubans, D. R. (2014). Fundamental movement skills and physical activity among children living in low-income communities: a cross-sectional study. International Journal of Behavioral Nutrition and Physical Activity, 11(1), 49-58.

Conde Caveda, J., y Viciana, V. (1997). Fundamentos para el desarrollo de la motricidad en edades tempranas. Archidona, España: Aljibe.

Coto, G. (2002). Análisis de la aplicación de la metodología matemáticas activa y creativa y su relación con la adquisición del proceso lógico operatorio, a nivel concreto en los niños y niñas de 5 y 6 años de los Jardines de Niños San Felipe de Alajuelita, El Rosario en Barrio Luján y la Divina Providencia en Paso Ancho (Tesis de licenciatura). Universidad Latina de Costa Rica. San José, Costa Rica.

Fairbrother, J. T. (2010). Fundamentals of Motor Behavior. Champaign, IL: Human Kinetics 1.

Fernández, D., Obando, J., Rodríguez, M., Salazar, E., y Trejos, M. (2007). Experiencia didáctica para la evaluación de conocimiento matemático y relaciones sociales de niños entre 5 y 6 años a partir de la interacción con materiales físicos y físicos digitales (Tesis de licenciatura). Universidad de Costa Rica. Ciudad Universitaria Rodrigo Facio. San José, Costa Rica.

Gallahue, D., y Ozmun, J. (2003). Compreendendo o desenvolvimiento motor: Bebês, crianças, adolescentes y adultos (2da ed.). São Paulo: Phorte Editora.

Gabbard, C. P. (2012). Lifelong Motor Development (6th ed.). San Francisco: Benjamin-Cummings Publishing Company.

Godino, J., Batanero C. y Font, V. (2003). Fundamentos de la enseñanza y el aprendizaje de las matemáticas para maestros. Recuperado de http://www.ugr.es/local/jgodino/edumat-maestros/

Gutiérrez, D., Bartolomé, R., y Hernán, L. (1997). Educación infantil II. Expresión y comunicación. Metodología del juego. Madrid: Mc Graw-Hill e Interamericana de España.

Hardy, L. L., Reinten-Reynolds, T., Espinel, P., Zask, A., \& Okely, A. D. (2012). Prevalence and correlates of low fundamental movement skill competency in children. Pediatrics, 130(2), e390-e398. http://doi.org/10.1542/ peds.2012-0345

Hernández, R., Fernández, C., y Baptista P. (2006). Metodología de la investigación (4ta ed,). México: McGraw- Hill Interamericana.

Lleixa, M. (2004). Necesidades motrices en la edad infantil. La educación física de 3 a 8 años (pp. 7-16). Barcelona, España: Paidotribo.

Medrano, G. (2001). El niño, su crecimiento. Aspectos motores, intelectuales, afectivos y sociales. Educación infantil: Descubrimiento de sí mismo y del entorno 0 -6 años (5ta ed.). Barcelona: Paidotribo.

Ministerio de Educación Pública. (1996). Programa de estudios de la educación preescolar. San José, Costa Rica: MEP.

Mira, M. (2001). Introducción al lenguaje matemático. Educación infantil: Expresión y comunicación 0 -6 años (5ta ed.). Barcelona: Paidotribo.

Molina, J. (1990). El juego, su importancia: Evolución en el ciclo inicial. Revista Comunidad Educativa, 26-31.

Núñez, F., López, K., Quesada, N., Pineda, V., y Solano M. (2008). La enseñanza de la resolución de problemas en el área de geometría en el II Ciclo de la Educación General Básica: Una propuesta metodológica (Tesis de licenciatura). Universidad De Costa Rica. Ciudad Universitaria Rodrigo Facio. San José, Costa Rica.

Oña, A. (1994). Comportamiento motor. Bases psicológicas del movimiento humano. Granada: Universidad de Granada.

Piaget, J. (1975). El nacimiento de la inteligencia del niño. Madrid: Aguilar.

Piaget, J. (1985). Seis estudios de psicología. Barcelona: Planeta Agostini.

Quirós, V.y Arráez, J. (2006). Juego y psicomotricidad. Propuesta y análisis de un programa de trabajo. Retos. Nuevas tendencias de educación física, deporte y recreación, 9, 23-31. 
Ruíz, A. (2000). El desafío de las matemáticas. Heredia, Costa Rica: Editorial de la Universidad Nacional.

Ruiz, F., García, A., Gutiérrez, F., Marqués, J., Román, R., y Samper, M. (2003). El juego y la motricidad en la etapa de la educación infantil. Los juegos en la motricidad infantil de los 3 a los 6 años. Barcelona, España: INDE.

Serrano, A., Azofeifa, A., y Araya, G. (diciembre 2008). Aprendizaje de las matemáticas por medio del movimiento. Una alternativa más de la educación física. Revista MHSalud, 5(2), 1-20. Recuperado de http:// www.redalyc.org/articulo.oa?id=237017536001

Sobalvarro L (2015). Geometría en movimiento: Efecto de su aplicación sobre el aprendizaje de la noción de objeto según la forma, para el ciclo de Transición de la Educación Preescolar (Tesis de maestría). Universidad de Costa Rica. San José Costa Rica.

Spessato, B., Gabbard, C., \& Valentini, N. C. (2013). The role of motor competence and body mass index in children's activity levels in physical education classes. Journal of Teaching in Physical Education, 32(2), 118-130.

Velázquez, A., y Martínez, A. (2005). Planteamientos teóricos. En Desarrollo de habilidades a través de materiales alternativos . Sevilla, España: Wanceulen.

Verlee, L. (1986). Aprender con todo el cerebro. Barcelona: Martínez Roca.

Viciana, V. (2000). Efectos de un programa metodológico lúdico para la mejora de los contenidos curriculares en niños/ as del segundo ciclo de la etapa de educación infantil (Tesis doctoral). Nicaragua: Universidad de Granada.

Wallon, H. (1978). Del acto al pensamiento. Buenos Aires: Psique.

\section{BY-NC-ND}

